\title{
POLARIZED ELECTRONS IN SURFACE PHYSICS
}


This page is intentionally left blank 


\section{POLARIZED}

\section{ELECTRONS IN}

SURFACE PHYSICS

R. Feder

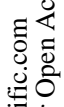

起

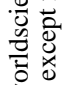

.

World Scientific 
Published by

World Scientific Publishing Co. Pte. Ltd.

P. O. Box 128, Farrer Road, Singapore 9128

Library of Congress Cataloging-in-Publication Data is available.

\section{Polarized Electrons in Surface Physics}

Copyright $\odot 1985$ by World Scientific Publishing Co. Pte. Ltd.

All rights reserved. This book, or parts thereof, may not be reproduced in any form or by any means, electronic or mechanical, including photo. copying, recording or any information storage and retrieval system now known or to be invented, without written permission from the Publisher.

ISBN 9971-978-49-0

9971-978-50-4 pbk

Printed in Singapore by Kim Hup Lee Printing Co. Pte. Ltd. 


\section{CONTENTS}

Foreword $\ldots \ldots \ldots \ldots \ldots \ldots \ldots \ldots \ldots \ldots \ldots \ldots \ldots \ldots \ldots \ldots$ xiii

\section{Part I: Theoretical Foundation}

1. Electronic and Magnetic Structure of Solid Surfaces

A. J. Freeman, C. L. Fu, S. Ohnishi \& M. Weinert . . . . . . . . 3

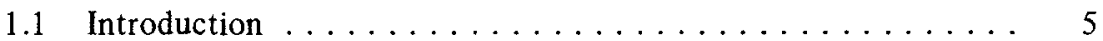

1.2 Theoretical Framework $\ldots \ldots \ldots \ldots \ldots \ldots \ldots$

1.2.1 Local Spin Density Functional Theory . . . . . . . . 9

1.2.2 Thin-Slab Approximation $\ldots \ldots \ldots \ldots \ldots \ldots \ldots \ldots . \ldots \ldots$

1.3 Approach and Methodology $\ldots \ldots \ldots \ldots \ldots \ldots \ldots \ldots \ldots$

1.3.1 FLAPW Method for Thin Films . . . . . . . . . . 13

1.3.2 Energetics of Surfaces: All-Electron Total

Energy Approach . . . . . . . . . . . . . . . . . 19

1.4 Magnetism of Transition Metal Surfaces $\ldots \ldots \ldots \ldots \ldots \ldots 20$

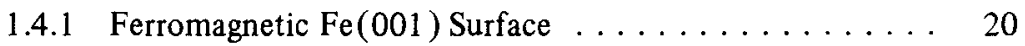

1.4.2 Ferromagnetic Ni(001) Surface $\ldots \ldots \ldots \ldots \ldots \ldots 25$

1.4.3 On the Possibility of Surface Magnetism V(001) .... . 29

1.4.4 Surface Magnetism of $\operatorname{Cr}(001) \ldots \ldots \ldots \ldots . \ldots . \ldots 35$

1.4.5 Induced Magnetism and Knight Shift of Pt(001) . . . . . 4 41

1.5 Magnetism at Bimetallic Interfaces . . . . . . . . . . 44

1.5.1 Magnetism at the $\mathrm{Ni} / \mathrm{Cu}$ Interface $\ldots \ldots \ldots \ldots \ldots . . \ldots 4$

1.5.2 Magnetism of the $\mathrm{Fe}(001)$ Surface Overlayered with $\mathrm{Ag} \ldots \ldots \ldots \ldots \ldots \ldots \ldots \ldots$

1.6 Effects of Adsorbates on Magnetic Surfaces:

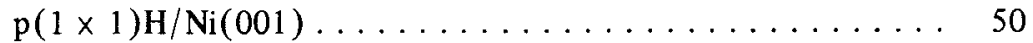

1.7 Two-Dimensional Magnetism of Metallic Overlayers, Interfaces and Superlattices $\ldots \ldots \ldots \ldots \ldots \ldots \ldots$

2. Ferromagnetism of Transition Metals at Finite Temperatures

H. Capellmann ... . . . . . . . . . . . . . . . . 67

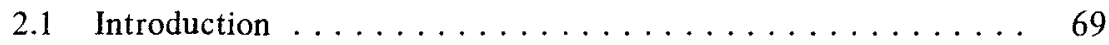

2.2 Finite Temperature Properties; What Drives the Phase

Transition? 
2.3 The Paramagnetic Phase $\ldots \ldots \ldots \ldots \ldots \ldots \ldots \ldots \ldots . \ldots 78$

2.4 Concluding Remarks $\ldots \ldots \ldots \ldots \ldots \ldots \ldots \ldots \ldots$

3. Critical Behaviour at Surfaces of Ferromagnets

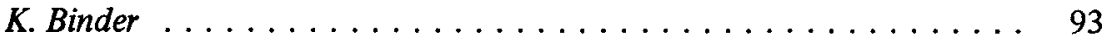

3.1 Introduction $\ldots \ldots \ldots \ldots \ldots \ldots \ldots \ldots \ldots \ldots \ldots$

3.2 Mean Field Theory for the Semi-Infinite Ising Model $\ldots \ldots \ldots 101$

3.3 Scaling Theory and Renormalization Group Results . . . . . . . 107

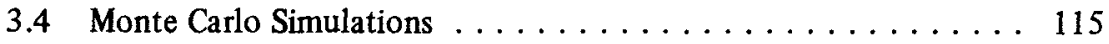

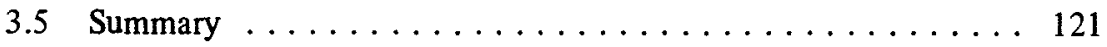

4. Principles and Theory of Electron Scattering and Photoemission

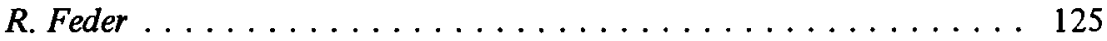

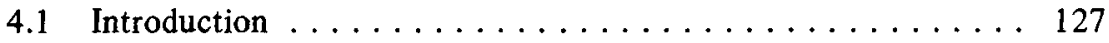

4.2 One-Electron Picture $\ldots \ldots \ldots \ldots \ldots \ldots \ldots \ldots \ldots \ldots$

4.2.1 Relativistic Electron-Ferromagnet Hamiltonian . . . . . 130

4.2.2 Effective One-Electron Potentials . . . . . . . . . 133

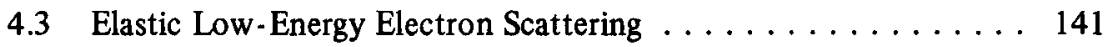

4.3.1 Basic Concepts . . . . . . . . . . . . . . . 141

4.3.2 Time Reversal and Spatial Symmetries . . . . . . . . 146

4.3.3 Single-Site Scattering $\ldots \ldots \ldots \ldots \ldots \ldots \ldots \ldots \ldots$

4.3.4 General Many-Site Scattering . . . . . . . . . . 156

4.3.5 Diffraction by Two-Dimensionally Periodic Systems . . . . 158

4.3.6 Disorder and Lattice Vibration Effects . . . . . . . . . 164

4.3.7 Determination of Magnetic and other Surface Properties . . 165

4.4 Inelastic Scattering and Secondary Electron Emission . . . . . . 170

4.4.1 Loss Mechanisms and Spin-Dependence . . . . . . . 171

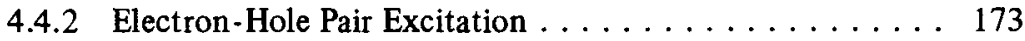

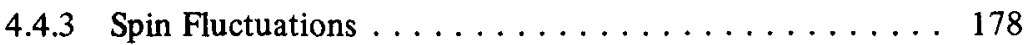

4.4.4 Imaginary Potential and Mean Free Path . . . . . . 181

4.4.5 True Secondary Electron Emission . . . . . . . . . . 183

4.5 Photoemission . . . . . . . . . . . . . . 185

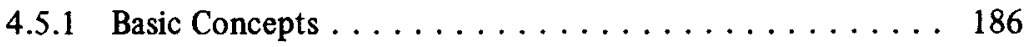

4.5.2 General Theoretical Framework . . . . . . . . . 188

4.5.3 Relativistic One-Step Theory for Ferromagnets . . . . . . 192

4.5.4 "Golden Rule" Form and Three-Step Model . . . . . . . 195

4.5.5 Nonrelativistic Limit and Electric Dipole Approximation . . 198 
4.5.6 Non-Magnetic Solids with Spin-Orbit Coupling . . . . . . 201 4.5.6.1 One-Step-Model Features and Selection Rules ... 201

4.5.6.2 Bulk Interband Transitions . . . . . . . . . . 214

4.5.6.3 Transport Effects . . . . . . . . . . 218

4.5.6.4 Surface Transmission . . . . . . . . . . . . . 222

4.5.6.5 Emission from Adsorbates . . . . . . . . . . . . 224

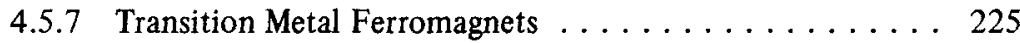

4.5.7.1 At Low Temperature . . . . . . . . . . . 226

4.5.7.2 Near the Curie Temperature . . . . . . . . . 228

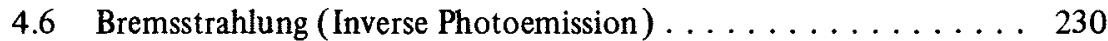

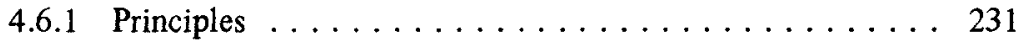

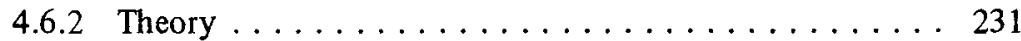

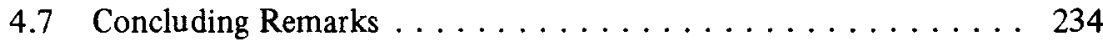

\section{Part II: Experiments and Results}

5. Sources and Detectors for Polarized Electrons

J. Kirschner . . . . . . . . . . . . . . . . . . . . . . . . . . 245

5.1 Sources of Polarized Electrons . . . . . . . . . . . . . . . 247

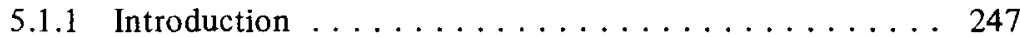

5.1 .2 Photoemission Sources . . . . . . . . . . . . . 248

5.1.3 The Field Emission Source . . . . . . . . . . . . . . 259

5.2 Detectors for Polarized Electrons . . . . . . . . . . . . . . . . 264

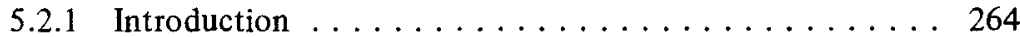

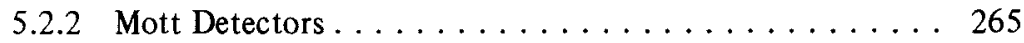

5.2.3 The Absorbed Current Detector . . . . . . . . . 271

5.2 .4 The LEED Detector . . . . . . . . . . . . . . . . 276

6. Elastic Spin-Polarized Low Energy Electron Diffraction from Non-Magnetic Surfaces

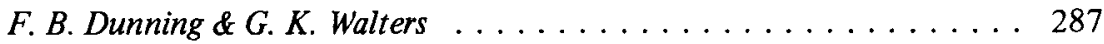

6.1 Introduction . . . . . . . . . . . . . . . . . . . . 289

6.2 Spin-Dependent Elastic Scattering - Basic Concepts . . . . . . . 290

6.3 Experimental Considerations . . . . . . . . . . . . . 294

6.4 Experimental Results and Comparisons with Theory . . . . . . 301

6.4 .1 Tungsten . . . . . . . . . . . . . . . 301

6.4.1.1 $\mathrm{w}(001)(1 \times 1) \ldots \ldots \ldots \ldots \ldots \ldots \ldots$

6.4.1.2 Adsorbate- and Temperature-Induced

Reconstruction . . . . . . . . . . . . . . . 304

6.4.1.3 Surface Barrier Resonances . . . . . . . . . . . 307 
6.4 .2 Gold . . . . . . . . . . . . . . . . . . 309

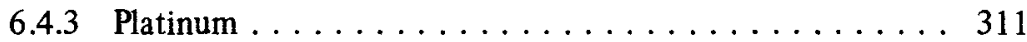

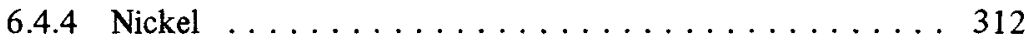

$6.4 .4 .1 \mathrm{Ni}(001) \ldots \ldots \ldots \ldots \ldots \ldots \ldots \ldots \ldots \ldots$

$6.4 .4 .2 \mathrm{Ni}(001) \mathrm{c}(2 \times 2) \mathrm{Te} \ldots \ldots \ldots \ldots \ldots \ldots . \ldots . \ldots 314$

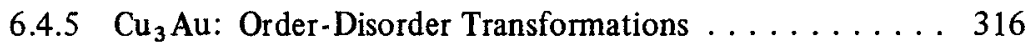

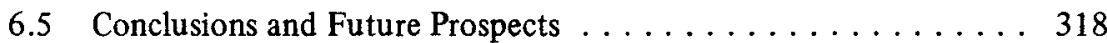

7. Elastic Spin-Polarized Low-Energy Electron Scattering from Magnetic Surfaces

U. Gradmann \& S. F. Alvarado . . . . . . . . . . . . . . . . 321

7.1 Introduction: Spin Polarized Electron Scattering in

Comparison with other Experimental Probes of Surface

Magnetism . . . . . . . . . . . . . . . . . . . 323

7.2 Surface Magnetization of 3d-Magnets at Low Temperatures . . . . 326

7.3 Surface Magnetism and Chemisorption at 3d-Magnet Surfaces . . . 334

7.4 Critical Behavior of Ni-Surfaces . . . . . . . . . . . . 338

7.5 Enhanced Surface Curie Temperature and Surface Magnetic

Reconstruction of $\mathrm{Gd}(0001) \ldots \ldots \ldots \ldots \ldots \ldots \ldots$

7.6 Ferromagnetic Glasses . . . . . . . . . . . . . . . . . 344

8. Inelastic Electron Scattering by Ferromagnets

J. Kirschner . . . . . . . . . . . . . . . . . . . . . . 353

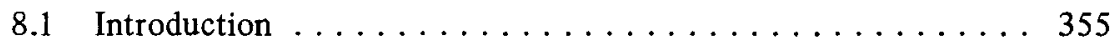

8.2 Spin-Dependence of the Electron Mean Free Path . . . . . 355

8.3 Two-Electron Scattering Processes . . . . . . . . . . . . 359

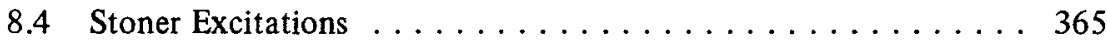

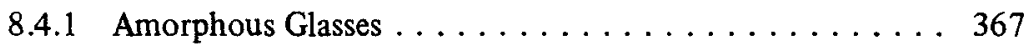

8.4 .2 Single Crystals $\ldots \ldots \ldots \ldots \ldots \ldots \ldots \ldots \ldots \ldots \ldots \ldots$

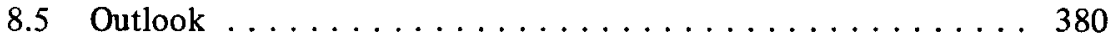

9. Spin Polarized Secondary Electron Emission from Ferromagnets

M. Landolt . . . . . . . . . . . . . . . . . . . . . 385

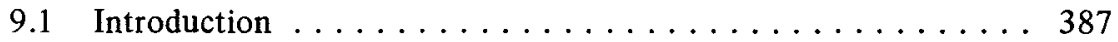

9.2 Spin Polarization of True Secondary Electrons . . . . . . . . . . . 389

9.2.1 Energy Distribution of the Secondary Electron Spin

Polarization . . . . . . . . . . . . . . . . . . . . 389

9.2.2 Magnetic Domain Microscopy . . . . . . . . . . . . . . . 394

9.2 .3 Magnetic Depth Profiling . . . . . . . . . . . . . . 397 
9.3 Spin Polarized Auger Spectroscopy . . . . . . . . . . . 402

9.3.1 The MMM Auger Process . . . . . . . . . . . . . . . . 403

9.3.2 The LMM Auger Process . . . . . . . . . . . . . . . . . 409

9.4 Electron Spin Polarization in Inner Shell Excitations in a Solid . . 412

9.5 Summary . . . . . . . . . . . . . . . . . . . 417

10. Spin Polarized Photoemission by Optical Spin Orientation in Semiconductors

F. Meier . . . . . . . . . . . . . . . . . . . . . . . . . . 423

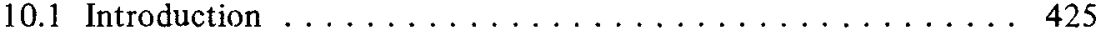

10.2 Principles of Optical Spin Orientation in Solids . . . . . . . . . 429

10.3 Applications of Optical Spin Orientation . . . . . . . . . 438

10.3.1 Hybridization of Energy Bands . . . . . . . . . 438

10.3.2 Lifting of the Spin-Degeneracy in Non-Centrosymmetric Materials . . . . . . . . . . . . . . . . . . . 445

10.3.3 Structural Phase Transitions . . . . . . . . . . . . 447

10.3.4 Enhancement of Resolution in Photoelectron Spectroscopy by Measuring the Spin Polarization . . . . . . . . . 452

10.3.5 Spin Exchange Scattering . . . . . . . . . . . 457

11. Spin-Resolved Photoemission from Nonmagnetic Metals and Adsorbates

U. Heinzmann \& G. Schönhense . . . . . . . . . . . . . . 467

11.1 Introduction . . . . . . . . . . . . . . . . . . . . 469

11.2 Energy -, Angle- and Spin-Resolved Photoelectron Emission

Technique . . . . . . . . . . . . . . 471

11.2.1 Experimental . . . . . . . . . . . . . . . 4 471

11.2.2 The "Complete" Photcionization Study . . . . . . . . . 474

11.3 Symmetry-Resolved Bandmapping of Pt(111) . . . . . . . 477

11.3.1 Relativistic Bandstructure ... . . . . . . . . . . . 477

11.3.2 Spin-Resolved Photoelectron Spectra and Bandmapping . . 478

11.3.3 Temperature Dependence . . . . . . . . . . . . . . 480

11.3.4 Comparison with Quantitative Theoretical Calculations . . 482

11.4 Hybridization Effects and Special Regions of Energy Bands . . . . 485

11.4.1 The W(100) Case . . . . . . . . . . . . . . 485

11.4.2 The $\Lambda$-Direction of $\mathrm{Pt}$ and $\mathrm{Au} \ldots \ldots \ldots \ldots \ldots . \ldots 487$

11.5 Off-Normal Photoelectron Emission . . . . . . . . . . 490

11.5.1 Surface Transmission Effects . . . . . . . . . . . . . 490

11.5.2 Dependence of the ESP Vector on the Emission Angle . . . 492 
11.6 Photoelectron Spinpolarization Spectroscopy of Physisorbed Rare Gases . . . . . . . . . . . . . . . . . . . . 495

11.6.1 Level Splitting . . . . . . . . . . . . . . . . . 495

11.6.2 Resonance Behavior of the Spin Polarization . . . . . . . 500

11.6.3 Influence of Substrate, Overlayer Structure and 2D

Phase Transitions . . . . . . . . . . . . . . . . . . . 504

11.7 Adsorbate-Induced Changes in Substrate ESP Spectra . . . . . . 506

12. Spin- and Angle-Resolved Photoemission from Ferromagnets

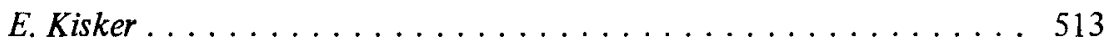

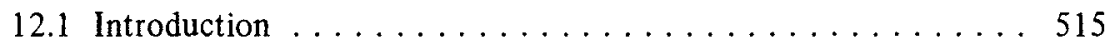

12.2 Experimental Set-Ups for Spin- and Angle-Resolved

Photoemission . . . . . . . . . . . . . . . . . 518

12.3 Experimental Results and Discussion $\ldots \ldots \ldots \ldots \ldots \ldots \ldots 21$

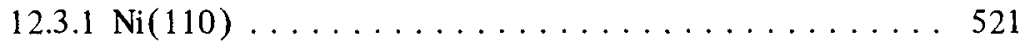

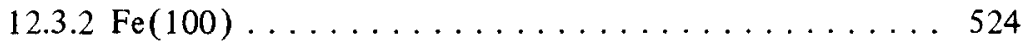

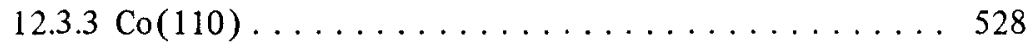

12.3.4 Oxygen Adsorption Studies . . . . . . . . . . . . 529

12.3.5 Auger Electrons and Resonant Photoemission from

3d-Transition Metals . . . . . . . . . . . . . . 530

12.3.6 Study of the Ferromagnetic to Paramagnetic Phase

Transitions of $\mathrm{Fe}$ and $\mathrm{Ni} \ldots \ldots \ldots \ldots 534$

12.3.6.1 Introduction . . . . . . . . . . . . 534

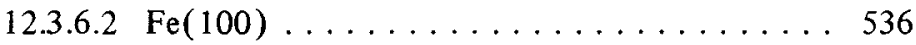

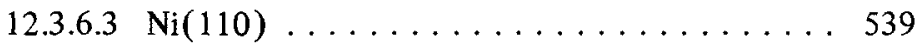

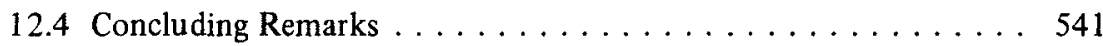

13. Spin Dependent Inverse Photoemission from Ferromagnets

V. Dose \& M. Glöbl . . . . . . . . . . . . . . . . . . . . . . 547

13.1 Introduction . . . . . . . . . . . . . . . . . 549

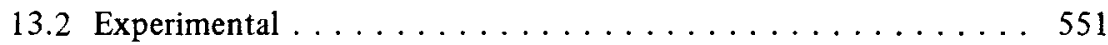

13.3 Room Temperature Data for Iron . . . . . . . . . . . 553

13.4 Measurements at Elevated Temperature ............ 559

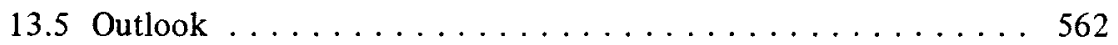

14. Photoemission and Bremsstrahlung from $\mathrm{Fe}$ and $\mathrm{Ni}$ :

Theoretical Results and Analysis of Experimental Data

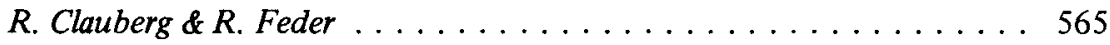

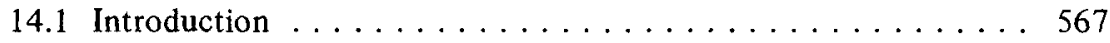


14.2 Analysis at Low Temperature $\ldots \ldots \ldots \ldots \ldots \ldots \ldots \ldots . \ldots 67$

14.2.1 $\mathrm{Fe}(001)$ and $\mathrm{Fe}(110) \ldots \ldots \ldots \ldots \ldots \ldots \ldots$

$14.2 .2 \mathrm{Ni}(110) \ldots \ldots \ldots \ldots \ldots \ldots \ldots \ldots \ldots \ldots \ldots \ldots$

$14.2 .3 \mathrm{Ni}(110)(2 \times 1)-\mathrm{O} \ldots \ldots \ldots \ldots \ldots \ldots \ldots . \ldots \ldots 3$

14.3 Analysis Near the Curie Temperature $\ldots \ldots \ldots \ldots \ldots \ldots \ldots 588$

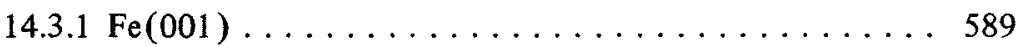

$14.3 .2 \mathrm{Ni}(110) \ldots \ldots \ldots \ldots \ldots \ldots \ldots \ldots \ldots . \ldots \ldots 7$

15. Polarized Electrons in Surface Physics: Outlook

M. Campagna . . . . . . . . . . . . . . . . . . . 605 
This page is intentionally left blank 


\section{Foreword}

Surface physics, dealing with physical (and thence also chemical) properties and processes related to the solid/vacuum boundary, has developed into a vast field of still growing scientific and technological importance. (An extensive survey and references may be found in four volumes recently edited by King and Woodruff (1981-1984)). Electrons are essential for surface physics in two respects: firstiy, bound electrons are a constituent part of any surface system, essential not only for the very existence of the system (its local bonding and geometry), but also for its vibrational, magnetic and chemical properties; secondly, free electrons provide - in a wide variety of scattering and emission techniques - powerful means of studying surface properties. Particularly interesting and useful phenomena occur, if the ensemble of electrons under consideration is polarized, i.e. if the number of electrons with spin parallel to a preferential direction differs from the number with spin antiparallel. For electrons bound to the surface system, this is associated with ferro- or ferrimagnetic ordering. In electron spectroscopy techniques, one is dealing with a beam of polarized electrons which is incident on the surface or emerges from it. (A general introduction to polarized free electrons and their use in atomic, solid state and high-energy physics is given in a monograph by Kessler (1976 and, updated, 1985)). Interacting with the surface system via exchange or spin-orbit coupling, polarized electron beams have - due to substantial advances in producing them ("sources") and analysing their spin polarization ("detectors") - within the past decade established themselves as a unique tool for studying magnetic, electronic and even geometrical surface properties.

In view of the achievements already made, the rapidly growing interest in and the future promise of this field, the time appears ripe for a comprehensive presentation, which initiates the non-specialist (with a general physics background at the graduate level) and 
reviews the current state of the art. This is the aim of the present book. It consists of a coherent sequence of fourteen chapters written by top level experts, who have significantly promoted progress in the respective sub-areas of the field.

The book is organized in two main parts: (I) theoretical foundation, (II) experiments and (experimental and theoretical) results.

Part I (Chapters 1 to 4 ) introduces fundamental concepts and theoretical approaches. Chapter 1 is devoted to the electronic and magnetic structure of clean and adsorbate-covered surfaces at temperatures well below their ferromagnetic transition temperature. After setting the theoretical framework of local spin-density-functional formalism and thin film approximation, the currently most fruitful first-principles theory for calculating the spin-polarized electronic ground state is introduced, and numerical results for a variety of typical transition metal surfaces and overlayers are presented and discussed. While ferromagnetism of the infinite solid ("bulk") at low temperatures is well understood (in particular itinerant Stoner model for 3d transition metals and Heisenberg Model for rare-earth systems) (cf. standard textbooks on Solid State Physics, e.g. Ashcroft and Mermin (1976), Callaway (1976), Harrison (1970)), transition metal ferromagnetism near the Curie temperature is a subject of very recent controversy and progress. Since an understanding of the "bulk" is a prerequisite for understanding the surface, and since polarized electrons (in photoemission) have provided valuable new insight, key concepts (spin fluctuations, local moments, short-range magnetic order) and theoretical state of the art are therefore included in the present book (Chapter 2). This is naturally followed by a survey of surface ferromagnetism near the Curie temperature (Chapter 3), i.e. "critical behaviour", including scaling theory, renormalization group and Monte Carlo simulations. Chapters 1 to 3 having dealt with the spin-polarized (ferromagnetic) structure of surface systems "by themselves", Chapter 4 addresses the interaction of polarized free electrons with magnetic and non-magnetic surface systems 
(semi-infinite solid with clean or adsorbate-covered surface), whicn is fundamental to a wide variety of electron scattering and emission techniques for investigating surface properties. Observable spin polarization effects may arise from ferromagnetic exchange interaction and/ or from spin-orbit coupling, which are both formally incorporated in a one-electron Dirac Hamiltonian containing an effective magnetic field. The theory of elastic spin-polarized low-energy electron diffraction (LEED) is presented in some detail, firstly because of its intrinsic importance and secondly because the "LEED state" (or its time reverse) is an essential ingredient for quantitative theories of other methods like photoemission, inverse photoemission and inelastic electron scattering, which are subsequently discussed. General results due to symmetry properties are presented, and principles of deducing, with the aid of theory, surface properties from experimental data are explained.

Part II (Chapters 5 to 14) deals with experimental techniques, experimental results and physical information obtained by comparing experimental data with their theoretical counterparts. Chapter 5 introduces the essential experimental tools: sources of polarized electrons, spin polarization detectors (polarimeters), synchrotron radiation (linearly and circularly polarized) and photon detectors. Elastic spinpolarized low-energy electron diffraction is presented for non-magnetic and for ferromagnetic surfaces in Chapters 6 and 7, respectively. In particular, the determination of the surface geometry, of the layerdependent-magnetization at low temperatures and of the ferromagnetic critical behaviour of surfaces is illustrated. The subsequent two Chapters are devoted to spin-dependent electron-electron collision processes. The techniques, which are presented, include in particular high-resolution electron energy loss spectroscopy from ferromagnets, culminating in a "triple scattering" experiment involving both a polarized primary beam and spin analys is of inelastically scattered electrons (Chapter 8), and secondary electron emission (especially Auger emission and the very-low-energy "cascade") (Chapter 9). In addition to revealing details of the electron-electron interaction and ferromagne- 
tic surface and bulk properties, these studies have led to the technologically important development of a magnetic scanning electron microscope. Chapters 10 to 12 are devoted to spin-resolved photoemission due to radiation in the (vacuum) ultraviolet range (photon energies up to about $70 \mathrm{eV}$ ). Chapter 10 focuses on semiconductor surfaces, for which spin-orbit coupling together with circular light polarization produces highly polarized photoelectrons, which carry information on the bulk band structure, on doping with impurities and - due to a most recently discovered spin precession effect in non-centrosymmetric crystals - on the spatial extent of the band-bending region near the surface. For non-magnetic metal surfaces, spin-, angle- and energy-resolved photoemission experiments performed with circularly polarized ultraviolet synchrotron radiation permit a direct observation of the symmetry types of the occupied states and promise detailed information on the electronic structure of adsorbed overlayers (Chapter 11). For ferromagnets (Chapter 12), spin-resolved photoemission by linearly polarized or unpolarized light reveals the majority- and minority-spin (quasi-particle) bulk and surface band structures. While photoemission observes the occupied electronic states, its inverse, bremsstrahlung induced by polarized electrons (Chapter 13), provides complementary information on the unoccupied states (in particular in the vicinity of the Fermi level). The retrieval of physical information from experimental photoemission and bremsstrahlung data by means of theoretical model calculations is illustrated in Chapter 14 for ferromagnetic Fe and $\mathrm{Ni}$. In particular, the determination of short-range magnetic order near the Curie temperature is demonstrated, and some light is shed on the influence of chemisorption on surface magnetism. The final Chapter 15 gives a synopsis and an outlook on future prospects for polarized electrons both in fundamental surface physics research and in technological applications.

The organization of Part II is such that physical properties of specific materials are presented in conjunction with the polarizedelectron method by which they were revealed. An alternative classifica- 
tion of the results according to materials is indicated in the following "cross reference" table, which may also serve as a Reader's guide to Chapters 6 to 14 .

\begin{tabular}{|c|c|c|c|c|c|}
\hline \multirow[b]{2}{*}{ Method } & \multicolumn{3}{|c|}{ ferromagnetic } & \multicolumn{2}{|c|}{ non-magnetic } \\
\hline & $\begin{array}{c}\text { meta } \\
3 \mathrm{~d}\end{array}$ & & $\begin{array}{l}\text { non- } \\
\text { metals }\end{array}$ & metals & $\begin{array}{l}\text { semi- } \\
\text { conductors }\end{array}$ \\
\hline elastic scattering & 7 & 7 & & 6 & \\
\hline inelastic scattering & 8 & 8 & & 8 & \\
\hline secondary emission & 9 & & 9 & 9 & \\
\hline photoemission & 12,14 & 12 & 12 & 11 & 10 \\
\hline bremsstrahlung & 13,14 & & & & \\
\hline
\end{tabular}

Table: Chapters, in which results for different types of material as obtained by polarized-electron methods are presented.

Having outlined the scope and contents of the present book, it seems pertinent to briefly mention some related methods for studying ferromagnetic surfaces, which have not been included. About a decade ago, a substantial research effort was devoted to field emission, i.e. to extracting polarized electrons from ferromagnets by applying a strong electric field (for reviews and references cf. Kessler 1976 and 1985, Campagna et a1. 1976, Feuchtwang et a1. 1978, Celotta and Pierce 1980). The development of spin-, angle- and energy-resolved photoemission (cf. Chapters 10-12) has, however, superseded field emission as a magnetic surface diagnostic technique, and the polarizedelectron source based on field emission from an EuS-coated $W$ tip has - despite its merits of about $90 \%$ polarization and high brightness not survived in the competition against the presently most widely used and even commercially available GaAs-photoemission source (cf. Chapter 5). Polarized field emission has therefore - to our knowledge - 
xviii

not been pursued further since about 1980, and belongs to the history of surface physics.

Since we concentrate on techniques involving free polarized electrons, tunneling between superconductors and ferromagnets (cf.e.g. Tedrow et al 1982, Feuchtwang et al. 1978), which yields information on the spin polarization of electron states very close to the Fermi energy, has not been included. For the same reason, Mössbauer spectroscopy (cf. review by Keune 1985) is not represented nor is another interesting nuclear-physics method known as "Electron Capture Spectroscopy" (cf. review by Rau 1982), in which deuterons impinging at grazing angles on a ferromagnetic surface pick up conduction electrons and thus carry information on surface magnetism. Another recent technique, which yet has to prove its quantitative merits, employs a polarized beam of low-energy positrons and measures the spin dependence of the positronium formation rate at the magnetic surface (Gidley et a1. 1982) (cf. also a monograph on positron studies: ed. Mills and Canter 1985). Further, an atomic-physics method, in which spin-polarized metastable atoms are ionized at a ferromagnetic surface and subsequently neutralized in a polarization-dependent conduction-band Auger process, appears promising (Onnelion et a1. 1984).

In conclusion of this Foreword, may we express a personal thought? The authors hope that the book is not only useful and enjoyable to you, dear Reader, but that you may also share some of their enthusiasm about this flourishing area of surface physics. 


\section{References}

Ashcroft N W and Mermin N D 1976 Solid State Physics (Holt, Rinehart and Winston, New York)

Callaway J 1976 Quantum Theory of the Solid State (Academic Press, New York)

Campagna M, Pierce D T, Meier F, Sattler K and Siegmann H C 1976 AdV. E1. and E1. Physics 41113

Celotta R J and Pierce D T 1980 in Adv. in Atomic and Molecular Physics 16101

Feuchtwang T E, Cutler P H and Schmit J 1978 Surface Sci.75 401

Gidley D W, Koymen A R and Capehart T W 1982 Phys. Rev. Lett. 491779

Harrison W A 1970 Solid State Theory (McGraw Hill, New York)

Kessler J 1976/1985 Polarized Electrons (Springer, Berlin Heidelberg New York)

Keune W 1985 in Proceedings of the International Conference on Applications of the Mößbauer Effect, Leuven, Sept. 1985, in "Hyperfine Interactions" (North Holland, Amsterdam)

King D A and Woodruff D P 1981-85 (ed) The Chemical Physics of Solid Surfaces and Heterogeneous Catalys is (North Holland, Amsterdam)

Mills A and Canter K F (ed) 1985 Positron Studies of Solids, Surfaces and Atoms (World Scientific Publishing Co, Singapore 1985)

Onellion M, Hart M W, Dunning F B and Walters G K 1984 Phys. Rev. Lett. 52380

Rau C 1982 J. Magn. Magn. Mater. 30141

Tedrow P M, Moodera J S and Meservey R 1982 Sol id State Commun. 44587 